\title{
Design and experimental analysis of nonreciprocal wave propagation in a space-time modulated beam
}

\author{
Emanuele Riva ${ }^{\mathrm{a}}$, Davide E. Quadrelli ${ }^{\mathrm{a}}$, Jacopo Marconi ${ }^{\mathrm{a}}$, Gabriele Cazzulani ${ }^{\mathrm{a}}$, and Francesco \\ Braghin $^{\mathrm{a}}$ \\ aPolitecnico di Milano, Department of Mechanical Engineering, Via La Masa 1, Milano 20156, \\ Italy
}

\begin{abstract}
Breaking reciprocity in wave propagation problems is of great interest within the research community, given the opportunity to design new devices for one-way communication with unprecedent performances. In the context of mechanics and phonon transport, directional wave manipulation can be achieved exploiting space-time periodic materials. Namely, structures whose elastic or physical properties are functions of space and time.

In this work we experimentally study nonreciprocal wave propagation in a space-time modulated beam based on piezoelectric actuation. The system is made of an aluminum beam with an array of piezoelectric patches bonded on its top and bottom surface. Each patch is attached to a negative capacitance (NC) shunt driven by switching circuit, which is able to provide a square-wave stiffness profile in time to the layered material. Spatiotemporal modulation is induced by phase shifting the temporal modulation law of three consecutive piezo-elements, generating a traveling Young's modulus that mimics the propagation of a wave along the beam dimension. As a result, we achieved $1 \mathrm{kHz}$ directional bandgaps, i.e. bandgaps at different frequencies for counter propagating waves, which can be moved in a range spanning 8-11 kHz.
\end{abstract}

Keywords: Nonreciprocal wave propagation; Mechanical diode; Space-time modulation; Phononic crystal.

\section{INTRODUCTION}

The principle of reciprocity in wave propagation problems states that, if a system is linear time-invariant (LTI), waves propagate from a point A to B in the same way they travel from B to A. The violation of such hypothesis is de facto a way to break the reciprocity principle and a method to design new devices that exhibit different propagation properties along apposite directions. Thus $(A \rightarrow B) \neq(A \leftarrow B)$, which also implies a break of time reversal symmetry (T-symmetry), i.e. the physical laws are no longer invariant under the transformation $T: t \rightarrow-t$. In analogy with the electrical counterpart, this effect is generally known as acoustic wave diode and is nowadays considered as an "hot topic" within the research community.

Several mechanisms have been investigated in the past in order to conceive and physically realize such class of devices. One possibility is to exploit nonlinear phenomena, ${ }^{1-3}$ such as internal resonances, ${ }^{4}$ metastability ${ }^{5}$ bifurcation and chaos ${ }^{6}$ which, on one hand, allow for a broadband nonreciprocal behaviors. On the other hand, their low-scale or on-chip implementation can be difficult to conceive and physically realize. This limitation results from the high displacements and forces that are often required in order to establish non-linear effects in the wave propagation.

An effective way to break the reciprocity principle is offered by space-time modulated materials. Compared to moving media and the aforementioned examples, this strategy relies on motionless structures with space-time dependent properties, which is very convenient from an engineering perspective. In this context, one can tailor the material's physical parameters to be periodic functions of space, which reflects in the formation of a bandgap in reciprocal domain. It is demonstrated that, upon addition of an appropriate temporal modulation, the gap central position shifts toward either lower or higher frequencies for waves propagating along opposite directions, therefore achieving one-way filtering effects. ${ }^{7}$

In mechanics, the study of nonreciprocal effects through spatiotemporal materials has been explored by Trainiti

Send correspondence to E. Riva

E-mail: emanuele.riva@polimi.it 
\& Ruzzene ${ }^{8}$ proposing a modified Plane Wave Expansion Method (PWEM). In this work, useful relationships between modulation's critical parameters and formation of nonreciprocal gaps are provided. In this context, the PWEM is also suitable for the analysis of $2 \mathrm{D}$ space-time varying membranes ${ }^{9}$ and discretely modulated materials. ${ }^{10,11}$ An alternative way to compute the band structure of space-time materials is offered by Finite Element Method (FEM) based procedures ${ }^{12}$ which, on one side is a more versatile approach and can be applied to a multitude of modulated materials. On the other hand, it provides less physical insight in the wave propagation behavior.

Even though several theoretical works have been proposed, the physical implementation of such logic can be very difficult to conceive and experimentally realize, especially in mechanics. For this reason, only few experimental demonstrations are nowadays present in the literature. Viable solutions have been recently explored by $\mathrm{C}$. Daraio's group by exploiting magneto-mechanical lattices ${ }^{13}$ or employing tunable magnetic resonators attached on otherwise plain beams. ${ }^{14}$ An alternative approach is proposed by Attarzadeh et. al. and consists in the exploitation of an array of rotating resonators ${ }^{15}$ placed on a passive beam. The non-axisymmetric moment of inertia of the resonator's beams generate traveling-like properties, which reflect on T-symmetry break. In acoustic, a similar behavior is obtained with space-time when time varying boundary conditions ${ }^{16}$ are applied to an otherwise passive acoustic channel.

All the aforementioned configurations rely on properly phase shifted modulation signals, which are able to control the effective parameters of consecutive sub-elements. This strategy allows to establish a traveling like profile which is responsible for a frequency bias in reciprocal space.

This study concerns the experimental realization of an elastic wave diode, which consists in an array of patches bonded on an otherwise plain beam. Each patch is connected to a NC circuit which is able to locally alter the effective sandwich stiffness, while temporal modulation is achieved through analog switches placed between a DC power supply and the active NC circuits. A traveling stiffness profile is established by phase-shifting consecutive time-modulated piezo-pairs, providing nonzero momentum to the medium and therefore generating directional gaps in the frequency-wavenumber domain.

\section{SYSTEM DESIGN}

We start considering a plain aluminum beam with $N=24$ pairs of piezoelectric patches of size $l_{p} \times h_{p} \times b=$ $22 \times 1 \times 20 \mathrm{~mm}$ bonded on the top and bottom surfaces and spaced by a distance of $l_{s}=2 \mathrm{~mm}$ between each other. The array of patches has a total length of $l=576 \mathrm{~mm}$ and is separated from left and right clamps by $l_{1}=690 \mathrm{~mm}$ and $l_{2}=1134 \mathrm{~mm}$ respectively, to minimize reflections during the wave propagation analysis. Each patch is connected to a Negative Capacitance (NC) shunt, which constitute an inherit feedback law able to decrease the effective stiffness of the piezoelectric material, according to the schematic in Fig. 1(a). Temporal modulation is practically determined switching between $\mathrm{ON}$ and $\mathrm{OFF}$ conditions the $\mathrm{NC}$ circuits, therefore alternating the effective sandwich stiffness between $E_{s, O N}$ and $E_{s, O F F}$ :

$$
\begin{aligned}
& E_{s, O N}=\frac{E_{A l} I_{A l}+2 E_{p}^{S U} I_{p}}{I_{A l}+2 I_{p}}, \\
& E_{s, O F F}=\frac{E_{A l} I_{A l}+2 E_{p} I_{p}}{I_{A l}+2 I_{p}},
\end{aligned}
$$

where:

$$
I_{A l}=\frac{b H^{3}}{12}, \quad I_{p}=\frac{b h_{p}^{3}}{12}+b h_{p}\left(\frac{H}{2}+\frac{h_{p}}{2}\right)^{2}
$$

$E_{p}$ and $E_{a l}$ are the piezo short circuit and substrate Young's moduli. Instead, the shunted Young's modulus $E_{p}^{S U}$ for a patch in 31 operational mode can be computed starting from the material's constitutive equations:

$$
\begin{aligned}
S_{1} & =s_{11}^{E} T_{1}+d_{31} E_{3} \\
D_{3} & =d_{31} T_{1}+\epsilon_{33}^{T} E_{3}
\end{aligned}
$$




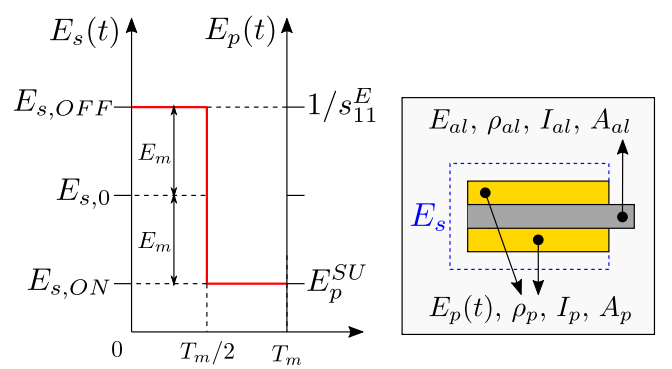

(a)

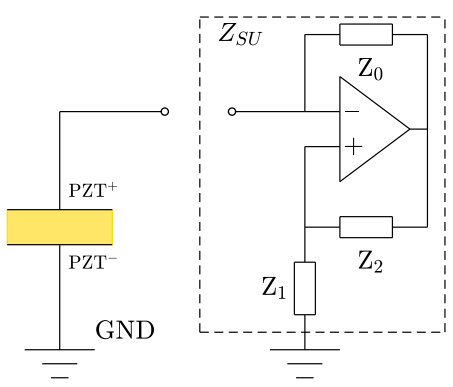

(b)

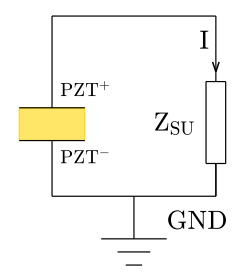

(c)

Figure 1. (a) Schematic of the sandwich stiffness modulation in time. A representation of the real unit cell parameters is shown alongside the modulation scheme. (b-c) Schematic of the shunted piezoelectric patch, along with a detailed view of the circuit.
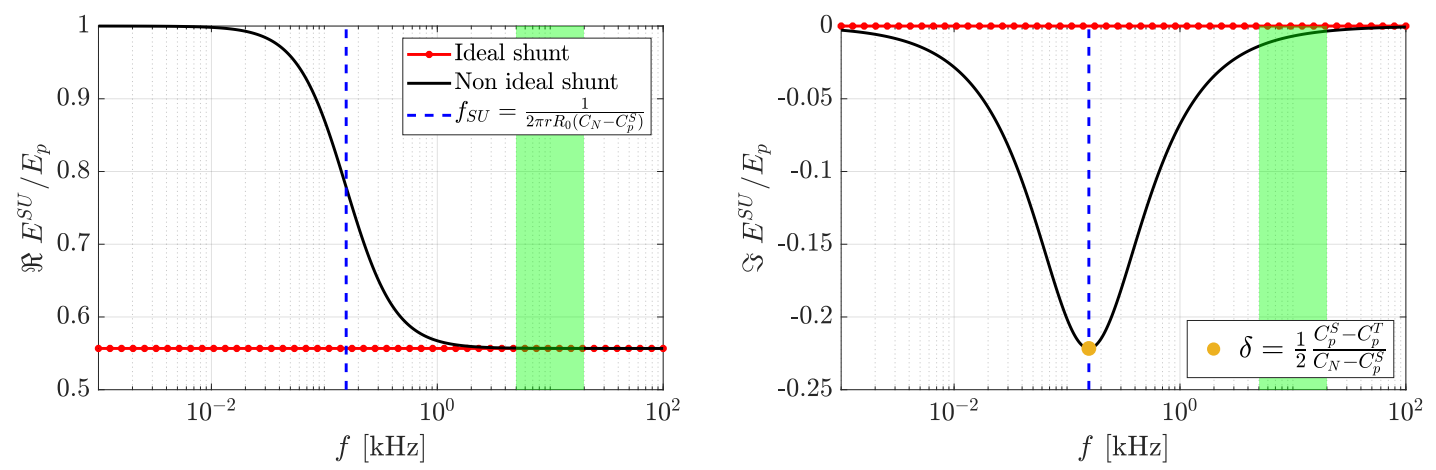

Figure 2. Comparison between ideal (red line) and non-ideal (black curve) effective shunted Young's modulus. Real part (left) and imaginary part (right). The vertical dashed line corresponds to the frequency pole of the system.

Considering the schematic in Fig. 1 (c), we can write the current balance of the circuit as:

$$
\frac{d}{d t} \int_{A} D_{3} d A=-\frac{V}{Z_{S U}}
$$

Combining Eqs. (2) and (3), one can write the following expression for the Young's modulus in Laplace domain:

$$
E_{p}^{S U}=\left[1-\frac{k_{31}^{2}}{1+s C_{p}^{T} Z_{S U}}\right] E_{p}
$$

where $C_{p}^{T}=\epsilon_{33} A / h_{p}$ is the piezoelectric capacitance and $k_{31}$ is the coupling coefficient. We now consider the negative impedance $Z_{S U}$ resulting from the circuit displayed in Fig 1(b):

$$
Z_{S U}=-\frac{Z_{1}}{Z_{2}} Z_{0}
$$

being $Z_{1}=R_{1}$ and $Z_{2}=R_{2}$ passive resistors, while $Z_{0}=\left(1 / R_{0}+s C_{0}\right)^{-1}$. The combination of Eqns. $4-5$ and enforcing $s=j \omega$ yields a compact expression for the frequency dependent Young's modulus:

$$
E_{p}^{S U}=\frac{1+\omega^{2} r^{2} R_{0}^{2}\left(C_{N}-C_{p}^{T}\right)\left(C_{N}-C_{p}^{S}\right)}{1+\omega^{2} r^{2} R_{0}^{2}\left(C_{N}-C_{p}^{S}\right)^{2}} E_{p}-j \omega r R_{0} \frac{\left|C_{p}^{s}-C_{p}^{T}\right|}{1+\omega^{2} r^{2} R_{0}^{2}\left(C_{N}-C_{p}^{S}\right)^{2}} E_{p} .
$$

that is characterized by a real part (effective stiffness modulation) and an imaginary part (negative loss factor) which, on one hand, can be suitably employed to favor lossless wave propagation. On the other hand it must be limited in amplitude in order to guarantee a global positive loss factor of the electromechanical system. This 


\begin{tabular}{cccc} 
Name & Value & Units & Description \\
\hline$R_{1}$ & 7.5 & $\mathrm{k} \Omega$ & - \\
\hline$R_{2}$ & 13.7 & $\mathrm{k} \Omega$ & - \\
\hline$R_{0}$ & 1000 & $\mathrm{k} \Omega$ & Bias resistance \\
\hline$C_{0}$ & 4.4 & $\mathrm{nF}$ & NC capacitance \\
\hline$C_{p}$ & 7 & $\mathrm{nF}$ & piezo patch capacitance \\
\hline$d_{31}$ & -1740 & $\mathrm{pm} / \mathrm{V}$ & piezo strain coefficient \\
\hline$k_{31}$ & 0.351 & - & piezo coupling coefficient \\
& Table 1. NC shunt circuit parameters.
\end{tabular}

non-ideal behavior is practically linked to the presence of a finite value of $R_{0}$, i.e. for $R_{0} \rightarrow \infty$ the imaginary part is nullified and the effective stiffness modulation writes:

$$
E_{p}^{S U}=\frac{C_{N}-C_{p}^{T}}{C_{N}-C_{p}^{S}} E_{p}
$$

A comparison between ideal and real behavior in frequency is illustrated in Fig. 2 for a value of $R_{0}=1 \mathrm{M} \Omega$, demonstrating that in the range of interest (highlighted in green) the circuit operates ideally. Interestingly, the circuits dynamic mainly affects low frequencies, while for frequencies higher than $f_{S U}=1 / 2 \pi R_{0}\left(C_{N}-C_{p}^{S}\right)$ (which is the electrical pole of shunt) the real dynamic behavior converges to the ideal case.

A spatiotemporal unit cell is constituted by three consecutive pairs of modulated patches, which are driven by phase shifted switching signals provided by Quad CMOS DG11B switches through the controller NI CompactRIO. The capability of the shunt to decrease the effective stiffness of the patch results in the following equivalent Young's modulus function:

$$
E_{s, k}(t)=E_{s, 0}\left[1+\alpha_{m} \operatorname{sign}\left(\cos \left(2 \pi f_{m} t+(k-1) \pi / 3\right)\right)\right]
$$

with $k=1,2,3$ denoting the sub-cell number, while $\pi / 3$ is the phase shift between the three consecutive active elements. $f_{m}=1 / T_{m}$ is the temporal switching frequency and $T_{m}$ the temporal period. Interestingly, positive and negative switching frequencies reflect on left or right traveling modulation profiles, which allow to mimic the propagation of a wave though the material and to induce nonreciprocal effects. ${ }^{8} \alpha_{m}=E_{m} / E_{s, 0}=27.5 \%$ is the achieved modulation amplitude, with $E_{s, 0}=\left(E_{s, O N}+E_{s, O F F}\right) / 2$ and $E_{m}=\left(E_{s, O N}-E_{s, O F F}\right) / 2$. In contrast, the equivalent material density is independent on time:

$$
\rho_{s}=\frac{\rho_{a l} A_{a l}+2 \rho_{p} A_{p}}{A_{a l}+2 A_{p}}
$$

being $\rho_{a l}=2700 \mathrm{Kg} / \mathrm{m}^{3}$ and $\rho_{p}=2700 \mathrm{Kg} / \mathrm{m}^{3}$ and $A_{a l}, A_{p}$ are the substrate and piezo cross-section area. Additional circuit parameters are listed in Table 1.

\section{EXPERIMENTAL RESULTS}

The wave propagation properties associated to the unit cell are investigated exploiting the PWEM presented in ref. ${ }^{10}$ applied to a Timoshenko beam model and compared to the experimental results, as shown in Fig. 3. In particular, it is experimentally and numerically demonstrated that the electroelastic beam supports a nonreciprocal bandgaps at approximately $8.5-10.5 \mathrm{kHz}$ for a switching frequency of $\pm 2 \mathrm{kHz}$. The experimental data are obtained imposing right traveling waves and changing the modulation's direction of propagation, which matches the expected results. Specifically, the system is forced using a wide spectrum tone burst excitation in correspondence of one of the clamps. The out of plane velocity field is measured in the modulated region by way of a Polytec 3D Scanner Laser Doppler Vibrometer (SLDV) and the dispersion relation $\hat{w}(\mu, f)$ is recovered through a $2 \mathrm{D}$ Fourier Transform (FT). $\mu=\kappa \lambda_{m}$ is the dimensionless wavenumber and $\lambda_{m}=3 l_{s}+3 l_{p}$ is the spatiotemporal unit cell size. The measurement, excitation and modulation are synchronized in order to assure each experiment is performed under the same condition, while consecutive tests are averaged in time, to decrease the noise. 

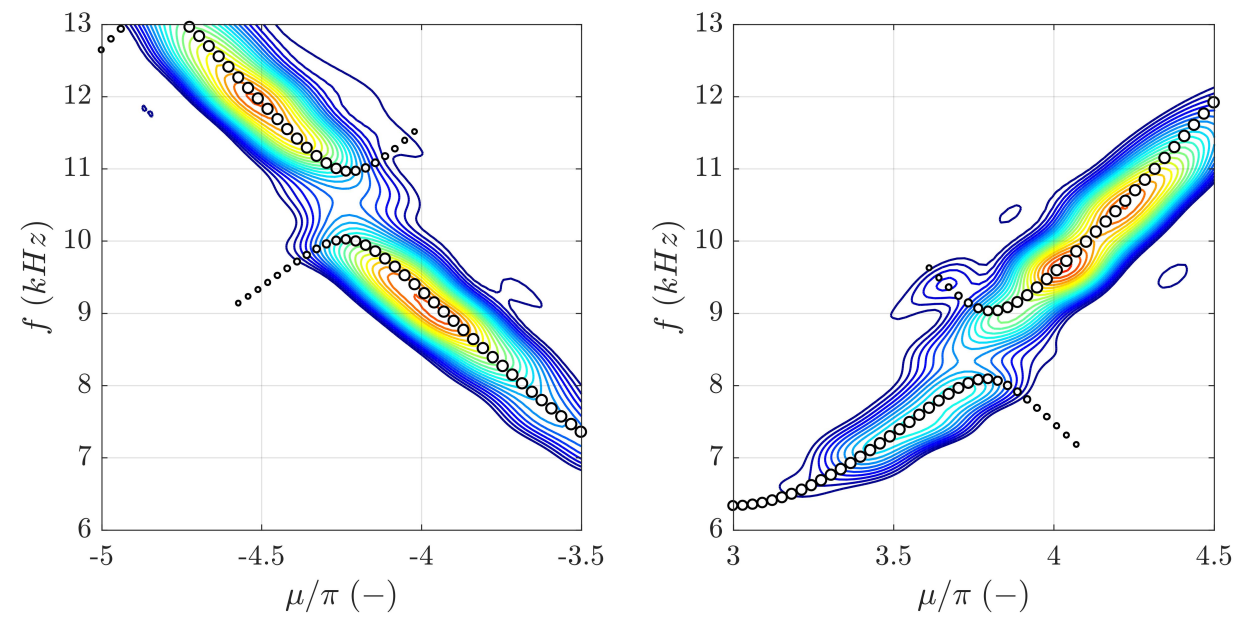

Figure 3. Comparison between experimental dispersion relation (colored contours) and numerical solution (white dots).

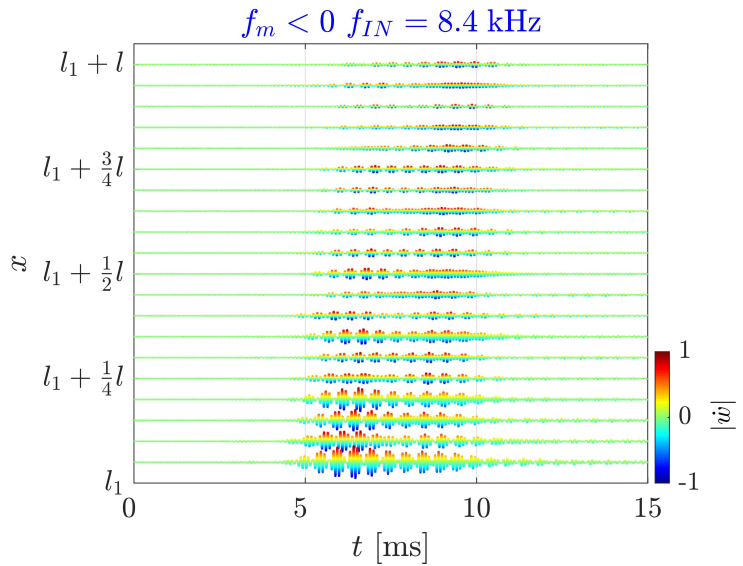

(a)

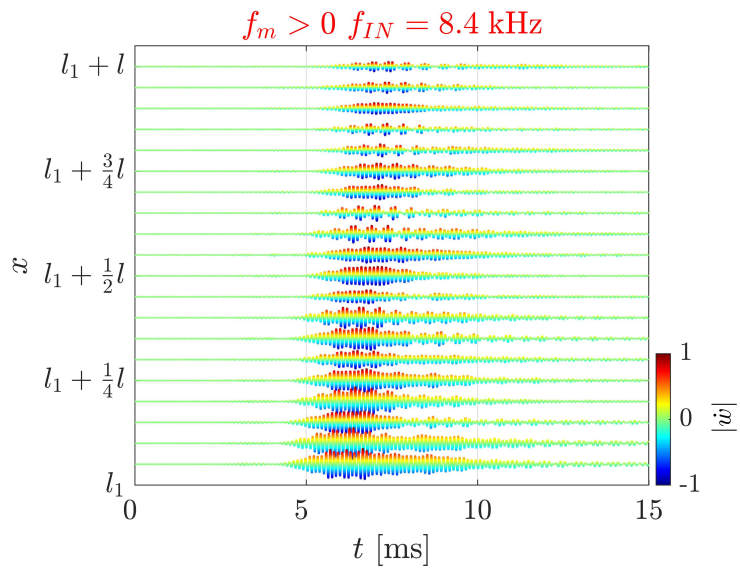

(c)

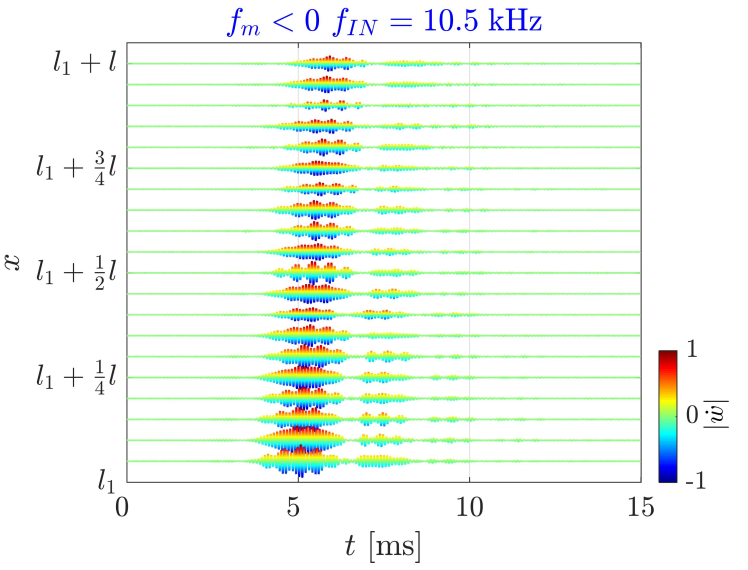

(b)

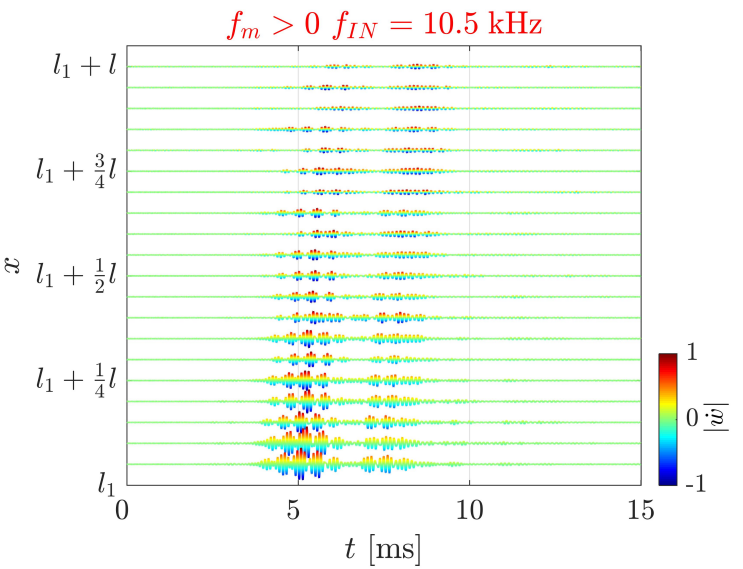

(d)

Figure 4. Waterfall plots for the modulated beam for excitation frequencies of 8.4 and $10.5 \mathrm{kHz}$ in case of $(\mathrm{a}-\mathrm{b}) f_{m}>0$ and (c-d) $f_{m}<0$. 


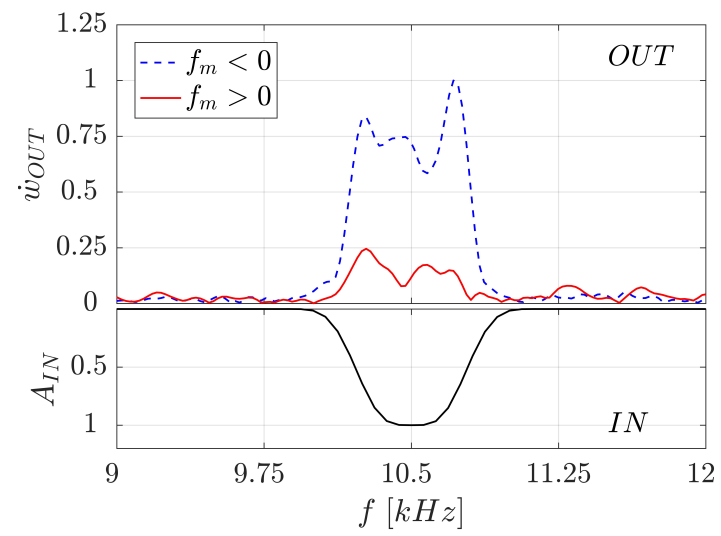

(a)

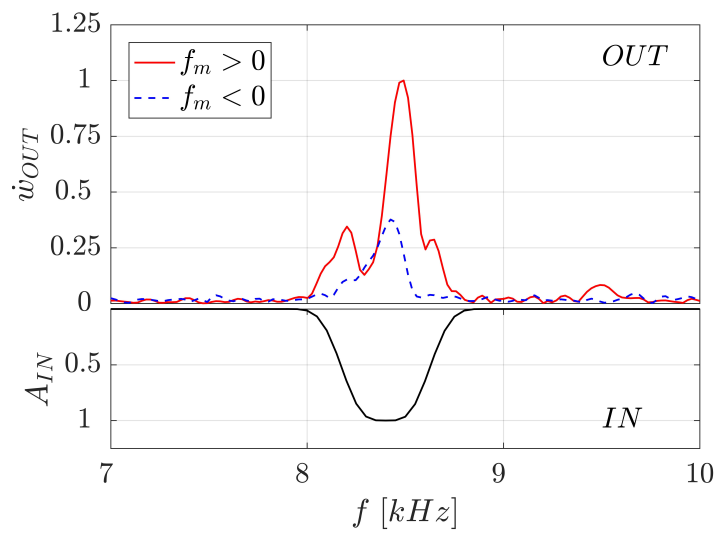

(b)

Figure 5. Spectrum of the signal exiting the modulated medium for a frequency of excitation (a) $f_{I N}=10.5 \mathrm{kHz}$ and (b) $f_{I N}=8.4 \mathrm{kHz}$. Dashed blue line is relative to $f_{m}<0$, while $f_{m}>0$ is represented with a red solid line. The input signal is displayed below the output spectrum.

Nonreciprocity is also tested exploiting a narrow spectrum tone burst excitation centered at the bandgap frequencies of $f_{I N}=8.4 \mathrm{kHz}$ and $f_{I N}=10.5 \mathrm{kHz}$ with a width of $\Delta f=1 \mathrm{kHz}$ approximately. The resulting time histories for negative switching frequency are reported in Fig. $4(\mathrm{a}-\mathrm{b})$ in terms of waterfall plots. Specifically, it is shown that for $f_{I N}=10.5 \mathrm{kHz}$ wave propagation is allowed, while, due to the presence of a nonreciprocal gap, waves are attenuated in space for an excitation frequency of $f_{I N}=8.4 \mathrm{kHz}$. A similar but opposite behavior is observed for positive switching frequencies as shown in Figs. 4(c-d) confirming that the system operates as a mechanical diode.

The performance of the electroelastic beam are further confirmed processing the signal exiting the modulated medium. The corresponding spectrum is displayed in Fig. 5(a-b) in which, positive and negative traveling modulation profiles $\left( \pm f_{m}\right)$ are compared for the same excitation frequency. It is worth noticing how, thanks to the directional filtering behavior, the system is able to perform one-way decay of approximately 1 order of magnitude.

\section{CONCLUSIONS}

In this work we have shown the design of an electroelastic beam with space-time varying properties able to break the reciprocity principle for elastic waves. In particular, stiffness modulation has been successfully realized by way of NC shunts, which are properly controlled in time to establish a traveling elasticity profile. This configuration allows for the formation of directional gaps that are here exploited to achieve one-way filtering.

The proposed setup can be easily integrated into micro electro-mechanical systems (MEMS), opening new possibilities for wave control in phononic communication devices.

\section{ACKNOWLEDGMENTS}

Support by the Italian Ministry for Education, University and Research through the project Department of Excellence LIS4.0 (Integrated Laboratory for Lightweight e Smart Structures) is also acknowledged.

\section{REFERENCES}

[1] Mojahed, A., Gendelman, O. V., and Vakakis, A. F., "Breather arrest, localization, and acoustic nonreciprocity in dissipative nonlinear lattices," The Journal of the Acoustical Society of America 146(1), $826-842$ (2019).

[2] Theocharis, G., Boechler, N., and Daraio, C., "Nonlinear periodic phononic structures and granular crystals," in [Acoustic Metamaterials and Phononic Crystals], 217-251, Springer (2013). 
[3] Fang, L., Darabi, A., Mojahed, A., Vakakis, A. F., and Leamy, M. J., "Broadband non-reciprocity with robust signal integrity in a triangle-shaped nonlinear 1d metamaterial," Nonlinear Dynamics , 1-13 (2020).

[4] Fronk, M. D., Tawfick, S., Daraio, C., Li, S., Vakakis, A., and Leamy, M. J., "Acoustic non-reciprocity in lattices with nonlinearity, internal hierarchy, and asymmetry: Computational study," Journal of Vibration and Acoustics 141(5) (2019).

[5] Wu, Z., Zheng, Y., and Wang, K., "Metastable modular metastructures for on-demand reconfiguration of band structures and nonreciprocal wave propagation," Physical Review E 97(2), 022209 (2018).

[6] Boechler, N., Theocharis, G., and Daraio, C., "Bifurcation-based acoustic switching and rectification," Nature materials 10(9), 665-668 (2011).

[7] Marconi, J., Cazzulani, G., Riva, E., and Braghin, F., "Observations on the behavior of discretely modulated spatiotemporal periodic structures," in [Active and Passive Smart Structures and Integrated Systems XII], 10595, 105952N, International Society for Optics and Photonics (2018).

[8] Trainiti, G. and Ruzzene, M., "Non-reciprocal elastic wave propagation in spatiotemporal periodic structures," New Journal of Physics 18(8), 083047 (2016).

[9] Attarzadeh, M. and Nouh, M., "Non-reciprocal elastic wave propagation in 2d phononic membranes with spatiotemporally varying material properties," Journal of Sound and Vibration 422, 264-277 (2018).

[10] Riva, E., Marconi, J., Cazzulani, G., and Braghin, F., "Generalized plane wave expansion method for non-reciprocal discretely modulated waveguides," Journal of Sound and Vibration 449, 172-181 (2019).

[11] Riva, E., Di Ronco, M., Elabd, A., Cazzulani, G., and Braghin, F., "Non-reciprocal wave propagation in discretely modulated spatiotemporal plates," Journal of Sound and Vibration, 115186 (2020).

[12] Vila, J., Pal, R. K., Ruzzene, M., and Trainiti, G., "A bloch-based procedure for dispersion analysis of lattices with periodic time-varying properties," Journal of Sound and Vibration 406, 363-377 (2017).

[13] Wang, Y., Yousefzadeh, B., Chen, H., Nassar, H., Huang, G., and Daraio, C., "Observation of nonreciprocal wave propagation in a dynamic phononic lattice," Physical review letters 121(19), 194301 (2018).

[14] Chen, Y., Li, X., Nassar, H., Norris, A. N., Daraio, C., and Huang, G., "Nonreciprocal wave propagation in a continuum-based metamaterial with space-time modulated resonators," Physical Review Applied 11(6), 064052 (2019).

[15] Attarzadeh, M., Callanan, J., and Nouh, M., "Experimental observation of nonreciprocal waves in a resonant metamaterial beam," Physical Review Applied 13(2), 021001 (2020).

[16] Karkar, S., De Bono, E., Collet, M., Matten, G., Ouisse, M., and Rivet, E., "Broadband nonreciprocal acoustic propagation using programmable boundary conditions: From analytical modeling to experimental implementation," Physical Review Applied 12(5), 054033 (2019). 